\title{
VORSCHAU 07-08 >
}

\section{ENTWICKLUNG}

\author{
Der neue Dreizylinder-TDI-Motor \\ von Volkswagen
}

Die neuen Drei- und VierzylinderDieselmotoren von BMW

\author{
Vom SULEV-Konzept der Abgasnach- \\ behandlung zur Steuerungssoftware \\ Aktiver Kraftstoffvorfilter für \\ Dieselmotoren im globalen Einsatz
}

\section{FORSCHUNG}

\section{Synthetischer Dieselkraftstoff OME1}

Schwerpunktbasierte Brennverlaufsvorausberechnung für homogen betriebene Ottomotoren

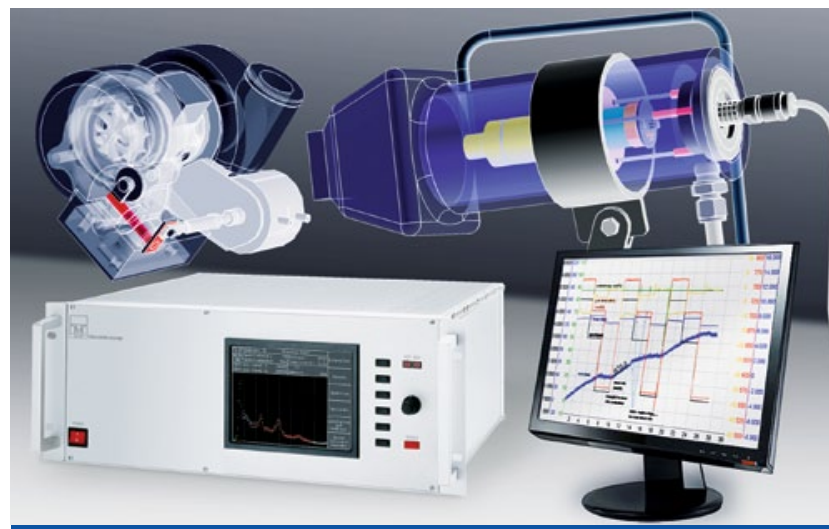

\section{TITELTHEMA}

\section{AUFLADUNG}

Die Rechnung ist ganz einfach: Ohne Aufladung gäbe es kein Motor-Downsizing und damit auch nicht den derzeit meistverfolgten Weg zur $\mathrm{CO}_{2}$-Reduzierung. Allerdings steigen mit höherer spezifischer Leistung des Motors auch die Anforderungen an die Aufladekomponenten und die Systemauslegung. Geblieben sind zudem die grundsätzlichen technischen Herausforderungen, die aus dem Zusammenwirken einer oder mehrerer Strömungsmaschinen und der Kolbenmaschine entstehen, wie die Anfahrschwäche bei geringen Motordrehzahlen.

Aufgaben gibt es also genügend für die Entwicklungsingenieure bei Motorherstellern, Zulieferern und Entwicklungsdienstleistern. In der nächsten MTZ stellen APL und IAVF Werkzeuge in Labor- und Prüfstandsbereichen für Entwicklung und Erprobung vor, mit deren Hilfe die mechanischen Komponenten des Aufladesystem auf die neuen Anforderungen ausgelegt werden können. FEV beleuchtet das Potenzial der elektrischen Zusatzaufladung durch höhere Bordnetzspannung - Stichwort $48 \mathrm{~V}$ unter verschiedenen Aspekten. Im Fokus stehen das Ansprechverhalten sowohl im Lastsprung als auch bei Fahrzeugbeschleunigung sowie Möglichkeiten im Stationärbetrieb und unter Fahrzyklusbedingungen.

Auch das Interview dreht sich um die elektrisch unterstützte Aufladung: Als einer der ersten OEMs hat sich Audi öffentlich für den künftigen Einsatz dieser Technik ausgesprochen. Mit Dr. Stefan Knirsch, Leiter der Motorentwicklung bei Audi, besprechen wir, welchen Weg der Hersteller dabei einschlagen wird. 